\title{
On the Evaluation of Integrals Related to the Error Function
}

\author{
By C. Chiarella and A. Reichel
}

1. Introduction. This paper presents some new methods of computing the functions

$$
\begin{aligned}
& U_{0}(x, t)=\frac{1}{(4 \pi t)^{1 / 2}} \int_{-\infty}^{\infty} \frac{e^{-(x-y)^{2 / 4} t} d y}{1+y^{2}}, \\
& V_{0}(x, t)=\frac{1}{(4 \pi t)^{1 / 2}} \int_{-\infty}^{\infty} \frac{e^{-(x-y)^{2 / 4 t}} y d y}{1+y^{2}}
\end{aligned}
$$

to a high degree of accuracy.

The function $U_{0}(x, t)$ has been tabulated by a number of authors [1], [2], [3], [4] and [5] (the latter authors also tabulate $V_{0}(x, t)$ ). The most accurate tabulation is that of Hummer [6].

Several methods of computing the related function $e^{z^{2}} \operatorname{erfc} z$ (see (1.5)) have been proposed, [7], [8] and [9]. Tabulations have been made for various regions in [10], [11], [12] and [13].

Of the methods presented below that in Section 2 has the advantage of being equally effective for all values of the arguments $x$ and $t$, while in those of Section 3 the terms of a series may be generated from recurrence relations after computation of the first two terms which involve no transcendental functions. Methods of computation for tabulation are also presented.

Before proceeding we note some well-known alternative forms of the functions. By taking the Fourier cosine and sine transforms of (1.1) and (1.2) respectively, and then inverting, we obtain the forms

$$
\begin{aligned}
& U_{0}(x, t)=\int_{0}^{\infty} e^{-p^{2} t-p} \cos p x d p, \\
& V_{0}(x, t)=\int_{0}^{\infty} e^{-p^{2} t-p} \sin p x d p,
\end{aligned}
$$

and hence we obtain

$$
W_{0}(x, t)=U_{0}(x, t)+i V_{0}(x, t)=\int_{0}^{\infty} e^{-p^{2} t-p(1-i x)} d p=\left(\frac{\pi}{4 t}\right)^{1 / 2} e^{w^{2}} \operatorname{erfc} w
$$

where

$$
w=(1-i x) / 2 t^{1 / 2} \text {. }
$$

From (1.1) and (1.2) by a simple change of variable we find

Received November 9, 1966. Revised June 22, 1967. 


$$
\begin{aligned}
W_{0}(x, t) & =\frac{1}{(4 \pi t)^{1 / 2}} \int_{-\infty}^{\infty} \frac{e^{-u^{2}} d u}{w+i u} \\
& =\frac{w}{(4 \pi t)^{1 / 2}} \int_{-\infty}^{\infty} \frac{e^{-u^{2}} d u}{u^{2}+w^{2}} \\
& =\frac{-i}{(4 \pi t)^{1 / 2}} \int_{-\infty}^{\infty} \frac{e^{-u^{2 / 4} t} d u}{u-(x+i)} .
\end{aligned}
$$

From (1.8), we derive the expressions

$$
\begin{aligned}
& U_{0}(x, t)=\frac{-i}{(4 \pi t)^{1 / 2}} \int_{-\infty}^{\infty} \frac{e^{-u^{2} / 4 t}(u-i) d u}{(u-i)^{2}-x^{2}}, \\
& V_{0}(x, t)=\frac{-x}{(4 \pi t)^{1 / 2}} \int_{-\infty}^{\infty} \frac{e^{-u^{2} / 4 t} d u}{(u-i)^{2}-x^{2}} .
\end{aligned}
$$

2. An Approximation Formula. A general method for evaluating integrals of the form

$$
\int_{-\infty}^{\infty} f(x) e^{-x^{2}} d x
$$

has been proposed by Goodwin [14]. We now apply this method to the form (1.7).

By the theorem of residues,

$$
\int_{C} \frac{e^{-z^{2}} d z}{\left(z^{2}+w^{2}\right)\left(1-e^{-2 \pi i z / h}\right)}=h \sum_{n=-\infty}^{\infty} \frac{e^{-n^{2} h^{2}}}{w^{2}+n^{2} h^{2}}+\frac{\pi e^{w^{2}}}{w\left(1-e^{2 \pi w / h}\right)}-\frac{\pi e^{w^{2}}}{w\left(1-e^{-2 \pi w / h}\right)}
$$

where $C$ is the rectangular contour $-R \leqq \operatorname{Re} z \leqq+R,|R| \rightarrow \infty,-\pi / h \leqq I z$ $\leqq+\pi / h$ and $h$ is sufficiently small for the contour to include the poles of the integrand at $z= \pm i w$.

Also

$$
\begin{aligned}
\int_{C} \frac{e^{-z^{2}} d z}{\left(z^{2}+w^{2}\right)\left(1-e^{-2 \pi i z / h}\right)}= & \int_{\infty+i \pi / h}^{-\infty+i \pi / h} \frac{e^{-z^{2}} d z}{\left(z^{2}+w^{2}\right)\left(1-e^{-2 \pi i z / h}\right)} \\
& +\int_{-\infty-i \pi / h}^{\infty-i \pi / h}\left\{\frac{e^{-z^{2}}}{z^{2}+w^{2}}+\frac{e^{-z^{2}-2 \pi i z / h}}{\left(z^{2}+w^{2}\right)\left(1-e^{-2 \pi i z / h}\right)}\right\} d z .
\end{aligned}
$$

Since

$$
\int_{-\infty-i \pi / h}^{\infty-i \pi / h} \frac{e^{-z^{2}} d z}{z^{2}+w^{2}}=\int_{-\infty}^{\infty} \frac{e^{-v^{2}} d v}{v^{2}+w^{2}}-\frac{\pi e^{w^{2}}}{w}
$$

we have

$$
\int_{C} \frac{e^{-z^{2}} d z}{\left(z^{2}+w^{2}\right)\left(1-e^{-2 \pi i z / h}\right)}=\int_{-\infty}^{\infty} \frac{e^{-v^{2}} d v}{v^{2}+w^{2}}-\frac{\pi e^{w^{2}}}{w}+E(h),
$$

where, after considerable manipulation, we find that

$$
E(h)=2 e^{-\pi^{2} / h^{2}} \int_{-\infty}^{\infty} \frac{e^{-z^{2}} d z}{\left\{(z-i \pi / h)^{2}+w^{2}\right\}\left\{1-e^{-2 \pi i z / h-2 \pi^{2} / h^{2}}\right\}} .
$$


Also

$$
\begin{aligned}
|E(h)| & \leqq 2 e^{-\pi^{2} / h^{2}} \int_{-\infty}^{\infty} \frac{e^{-z^{2}} d z}{1-e^{-2 \pi i z / h-2 \pi^{2} / h^{2}}} \\
& =2 e^{-\pi^{2} / h^{2}} \int_{-\infty}^{\infty} e^{-z^{2}}\left\{1+e^{-2 \pi i z / h-2 \pi^{2} / h^{2}}+\cdots\right\} d z
\end{aligned}
$$

so that

$$
\left.|E(h)| \leqq \frac{2 \sqrt{ } \pi e^{-\pi^{2} / h^{2}}}{1-e^{-\pi^{2} / h^{2}}} \quad \text { (e.g. for } h=\frac{1}{2}, E(h) \leqq 10^{-15}\right) .
$$

On regrouping terms we have

$$
\begin{aligned}
U_{0}(x, t)+i V_{0}(x, t)= & \frac{h}{w(4 \pi t)^{1 / 2}}+\frac{2 h w}{(4 \pi t)^{1 / 2}} \sum_{n=1}^{\infty} \frac{e^{-n^{2} h^{2}}}{w^{2}+n^{2} h^{2}} \\
& +\frac{\pi e^{w^{2}}}{(\pi t)^{1 / 2}\left(1-e^{2 \pi w / h}\right)}-\frac{w}{(4 \pi t)^{1 / 2}} E(h) .
\end{aligned}
$$

On separating real and imaginary parts and ignoring the error term we obtain

$$
\begin{aligned}
U_{0}(x, t) \approx & \frac{h}{\sqrt{ } \pi\left(1+x^{2}\right)}+\frac{h}{\sqrt{ } \pi} \sum_{n=1}^{\infty} \frac{e^{-n^{2} h^{2}}\left(1+x^{2}+4 t n^{2} h^{2}\right)}{\left(1-x^{2}+4 \operatorname{tn}^{2} h^{2}\right)^{2}+4 x^{2}} \\
& +\frac{A\left(\cos x / 2 t-e^{\eta} \cos \xi\right)}{1-2 e^{\eta} \cos \eta x+e^{2 \eta}}, \\
V_{0}(x, t) \approx & \frac{x h}{\sqrt{ } \pi\left(1+x^{2}\right)}-\frac{2 x h}{\sqrt{ } \pi} \sum_{n=1}^{\infty} \frac{e^{-n^{2} h^{2}}\left(1+x^{2}-4 \operatorname{tn}^{2} h^{2}\right)}{\left(1-x^{2}+4 \operatorname{tn}^{2} h^{2}\right)+4 x^{2}} \\
& +\frac{A\left(-\sin x / 2 t+e^{\eta} \sin \xi\right)}{1-2 e^{\eta} \cos \eta x+e^{2 \eta}},
\end{aligned}
$$

where $A=\sqrt{ } \pi e^{\left(1-x^{2}\right) / 4 t} / \sqrt{ } t, \eta=\pi / h \sqrt{ } t$ and $\xi=x(1 / 2 t-\eta)$.

It is found that the formulae (2.2) and (2.3) require less computation than the method of Salzer [8], which is equivalent to the method given by Abramowitz and Stegun [15].

Bounds on the error $E(h)$ are shown in the following table:

\begin{tabular}{cccccc}
\hline$h$ & 1 & 0.8 & 0.75 & 0.6 & 0.5 \\
\hline$E(h)$ & $10^{-4}$ & $10^{-6}$ & $10^{-7}$ & $10^{-11}$ & $10^{-15}$ \\
\hline
\end{tabular}

If, say, eight-place accuracy were required we would choose $h=0.7$ and would require eight terms in the infinite series in (2.2) and (2.3). The bounds on the error term are independent of the arguments $x$ and $t$. It is worth noting that the last term in (2.2), (2.3) may be ignored for $x / 2 t^{1 / 2}$ large, owing to the exponential term, e.g., for $x / 2 t^{1 / 2} \geqq 5$ this term may be neglected with an error of order $10^{-12}$. Suitable substitution in a formula given by Luke [19] for the function erfc (az) yields the first two terms of our equation (2.1). Luke's method of obtaining the error 
term is different. In Luke's work the error is effectively a combination of the last two terms of (2.1), and his error term in our context depends on the variables $x$ and $t$.

3. Derivatives of $U_{0}(x, t), V_{0}(x, t)$ and Methods of Computation. We shall use the notation

$$
\begin{aligned}
U_{n}(x, t) & =\partial^{n} U_{0}(x, t) / \partial x^{n}, \quad V_{n}(x, t)=\partial^{n} V_{0}(x, t) / \partial x^{n}, \\
W_{n}(x, t) & =\partial^{n}\left[U_{0}(x, t)+i V_{0}(x, t)\right] / \partial x^{n}=U_{n}(x, t)+i V_{n}(x, t) .
\end{aligned}
$$

We see from (1.3) and (1.4) that

$$
W_{n}(x, t)=i^{n} \int_{0}^{\infty} p^{n} e^{-p^{2} t-p+i p x} d p
$$

Integrating by parts, we get

$$
W_{n}(x, t)=-(x+i) W_{n-1}(x, t) / 2 t-(n-1) W_{n-2}(x, t) / 2 t,
$$

so that, on separating real and imaginary parts, we obtain the following recurrence relations for $U_{n}(x, t)$ and $V_{n}(x, t)$ :

$$
\begin{aligned}
& U_{n}(x, t)=\frac{-x U_{n-1}(x, t)}{2 t}+\frac{1}{2 t} V_{n-1}(x, t)-\frac{(n-1)}{2 t} U_{n-2}(x, t), \\
& V_{n}(x, t)=\frac{-U_{n-1}(x, t)}{2 t}-\frac{x}{2 t} V_{n-1}(x, t)-\frac{(n-1)}{2 t} V_{n-2}(x, t) .
\end{aligned}
$$

It is easily seen from (3.1) that

$$
\begin{aligned}
& U_{1}(x, t)=\left(V_{0}(x, t)-x U_{0}(x, t)\right) / 2 t \\
& V_{1}(x, t)=1 / 2 t-\left\{U_{0}(x, t)+x V_{0}(x, t)\right\} / 2 t .
\end{aligned}
$$

These recurrence relations (3.3) and (3.4) are essential for setting up recurrence relations for terms in series expansions of $U_{0}(x, t)$ and $V_{0}(x, t)$.

The polynomials $v_{n}(x, \alpha),[16]$, are defined by

$$
v_{n}(x,-\alpha)=\alpha^{n / 2} H_{n}\left(x /(4 \alpha)^{1 / 2}\right)
$$

where $H_{n}(x)$ denotes the Hermite polynomial. The $v_{n}(x, \alpha)$ have the orthogonality condition

$$
\int_{-\infty}^{\infty} e^{-x^{2} / 4 \alpha} v_{n}(x,-\alpha) v_{m}(x,-\alpha) d x=(4 \pi \alpha)^{1 / 2} \alpha^{n} 2^{n} n ! \delta_{m, n}
$$

It is convenient to seek an expansion of $W_{0}(x, t)$ in terms of these polynomials in the form

$$
W_{0}(x+y, t)=\sum_{n=0}^{\infty} A_{n} v_{n}(x,-\alpha) .
$$

Thus, using the orthogonality condition we have

$$
(4 \pi \alpha)^{1 / 2} \alpha^{n} 2^{n} n ! A_{n}=\int_{-\infty}^{\infty} e^{-x^{2} / 4 \alpha} v_{n}(x,-\alpha) W_{0}(x+y, t) d x .
$$

Using the result 


$$
v_{n}(x,-\alpha)=(-1)^{n} 2^{n} \alpha^{n} e^{x^{2 / 4 \alpha}} \partial^{n}\left(e^{-x^{2} / 4 \alpha}\right) / \partial x^{n}
$$

we find that

$$
\begin{aligned}
(4 \pi \alpha)^{1 / 2} n ! A_{n} & =(-1)^{n} \int_{-\infty}^{\infty} \frac{\partial^{n}}{\partial x^{n}}\left(e^{-x^{2} / 4 \alpha}\right) W_{0}(x+y, t) d x \\
& =\int_{-\infty}^{\infty} W_{n}(x+y, t) e^{-x^{2} / 4 \alpha} d \alpha, \quad \alpha>0
\end{aligned}
$$

Using (3.1) we then obtain,

$$
(4 \pi \alpha)^{1 / 2} n ! A_{n}=\imath^{n} \int_{0}^{\infty} p^{n} e^{-p^{2} t+i p y-p} d p \int_{-\infty}^{\infty} e^{i p x-x^{2 / 4 \alpha}} d x,
$$

which leads to the expression

$$
\begin{aligned}
A_{n} & =\frac{i^{n}}{n !} \int_{0}^{\infty} p^{n} e^{-p^{2}(t+\alpha)-p+i p y} d p \\
& =\frac{W_{n}(y, t+\alpha)}{n !} .
\end{aligned}
$$

Hence we arrive at the result

$$
W_{0}(x+y, t)=\sum_{n=0}^{\infty} \frac{v_{n}(x,-\alpha) W_{n}(y, t+\alpha)}{n !},
$$

or

$$
W_{0}(x+y, t-\alpha)=\sum_{n=0}^{\infty} \frac{v_{n}(x,-\alpha) W_{n}(y, t)}{n !} .
$$

By varying the parameters $x, y, t, \alpha$ in (3.5) and (3.6) we obtain a number of well-known expansions.

If $\alpha=1 / 4, y=0$ in (3.5) the Hermite expansion is obtained [17].

If $\alpha=0, y=0$, and using $v_{n}(x, 0)=x^{n}$, a Maclaurin expansion in $x$ is recovered, viz.,

$$
W_{0}(x, t)=\sum_{n=0}^{\infty} \frac{W_{n}(0, t) x^{n}}{n !} .
$$

If $x=i, y=X-i, \alpha=0$ in (3.6) we find that

$$
W_{0}(X, t)=\sum_{n=0}^{\infty} \frac{i^{n} W_{n}(X-i, t)}{n !},
$$

with

$$
W_{0}(X-i, t)=\frac{\sqrt{ } \pi}{2 t^{1 / 2}} e^{-X^{2 / 4 t}}+i e^{-X^{2}} \int_{0}^{X} e^{u^{2}} d u .
$$

Hummer [6], obtains essentially (3.7) by expanding the factor $e^{-p}$ in (1.3) and has used it to evaluate $U_{0}(x, t)$.

On putting $x=i+X, y=X-i, \alpha=0$ in (3.5) we obtain the Maclaurin expansion reported previously by the present authors [18] viz., 


$$
U_{0}(x, t)=\sum_{n=0}^{\infty} u_{n}, \quad V_{0}(x, t)=\sum_{n=0}^{\infty} v_{n},
$$

where the $u_{n}$ and $v_{n}$ are generated by

$$
\begin{aligned}
& u_{0}=\sqrt{ } \pi / 2 t^{1 / 2}, \quad v_{0}=0, \\
& u_{1}=-1 / 2 t, \quad v_{1}=x / 2 t, \\
& u_{n}=\left(\left(1-x^{2}\right) u_{n-2}+2 x v_{n}\right) / 2 t n, \\
& v_{n}=\left(\left(1-x^{2}\right) v_{n-2}-2 x u_{n-2}\right) / 2 t n .
\end{aligned}
$$

This procedure has the advantage that it is not necessary to generate any auxiliary function as is the case in many well-known methods (such as the error function) in order to start the computation.

The following scheme was used by the authors to construct a FORTRAN computer routine to evaluate $U_{0}(x, t)$ and $V_{0}(x, t)$ to 12 decimal places.

Use the Maclaurin expansion (3.8) for $x / 2 t^{1 / 2} \leqq 2$, and the approximation formulae (2.2) and (2.3) for $2 \leqq x / 2 t^{1 / 2} \leqq 7$, ignoring the trigonometric terms for $x / 2 t^{1 / 2}>5$.

For $x / 2 t^{1 / 2}>7$, use the asymptotic formula reported by the authors previously [19], viz.,

$$
\begin{aligned}
u_{0} & =-1, \quad u_{1}=-1 / x \\
u_{n} & =u_{n-1} / x-2 t(n-1) u_{n-2} / x^{2} \\
U_{0}(x, t) & \sim 1 / x \sum_{n=1}^{\infty}(-1)^{n} u_{2 n-1}, \quad V_{0}(x, t) \sim \frac{-1}{x} \sum_{n=0}^{\infty}(-1)^{n} u_{2 n} .
\end{aligned}
$$

4. Step-by-Step Evaluation. It is often required to evaluate $U_{0}(x, t)$ and $V_{0}(x, t)$ not just at one particular point but at a series of points. To use the methods described in Section 3 for this purpose would involve unnecessary computation and it has been found more convenient to use the Taylor expansions in the form described below.

Put $y=\Delta x$ and $\alpha=0$ in (3.5) to obtain

$$
W_{0}(x+\Delta x, t)=\sum_{n=0}^{\infty} \frac{(\Delta x)^{n} W_{n}(x, t)}{n !} .
$$

Now put $x=0, y=x, \alpha= \pm \Delta t$ and use the results

$$
v_{2 n}(0, t)=(2 n) ! t^{n} / n !, \quad v_{2 n+1}(0, t)=0,
$$

and we find that

$$
W_{0}(x, t \pm \Delta t)=\sum_{n=0}^{\infty} \frac{( \pm 1)^{n}(\Delta t)^{n} W_{2 n}(x, t)}{n !} .
$$

On separating real and imaginary parts we obtain formulae for $U_{0}(x, t)$ and $V_{0}(x, t)$, viz.,

$$
U_{0}(x+\Delta x, t)=\sum_{n=0}^{\infty} \frac{(\Delta x)^{n} U_{n}(x, t)}{n !}, \quad V_{0}(x+\Delta x, t)=\sum_{n=0}^{\infty} \frac{(\Delta x)^{n} V_{n}(x, t)}{n !}
$$




$$
\begin{aligned}
& U_{0}(x, t \pm \Delta t)=\sum_{n=0}^{\infty} \frac{( \pm 1)^{n}(\Delta t)^{n} U_{2 n}(x, t)}{n !}, \\
& V_{0}(x, t \pm \Delta t)=L_{n=0}^{\infty} \frac{( \pm 1)^{n}(\Delta t)^{n} V_{2 n}(x, t)}{n !} .
\end{aligned}
$$

The use of the recurrence relations (3.3) and (3.4) makes (4.3) and (4.4) relatively simple to use.

For instance, we could evaluate the functions for $t=10$ and $x=10$ (1) 20, say, by using (4.3). The initial values of the recurrence relations at any point are obtained from the value of the functions at the previous point.

With $\Delta x=1$, Eq. (4.3) has been used to obtain a table of eight significant figures for $x / 2 t^{1 / 2}<10$. It is necessary to regenerate the function afresh (by methods of Section 3) at $x / 2 t^{1 / 2}=5$ in order to maintain the stated accuracy. Convergence is fairly rapid; about 10 terms or less are required for $t>10$.

Similarly, we may proceed stepwise in $t$ using (4.4). In this case, in order to maintain accuracy over an appreciable range of $t$ it is necessary to start at a large value of $t$ and proceed back to the line $t=0$, (i.e., take $\Delta t$ negative). Convergence is again fairly rapid for $t>10,15$ terms and less in order to achieve eight significant figures. However, as $t$ approaches 1 , the number of terms required make it advisable to use the methods of Section 3.

School of Mathematics

Wollongong University College

Wollongong, N.S.W., Australia

Department of Applied Mathematics

The University of Sydney

Sydney, N.S.W., Australia

1. D. Posener, Austral. J. Phys., v. 12, 1959, pp. 184-196.

2. G. D. Finn \& D. Mugglestone, Monthly Notices Roy. Astronom. Soc., 129, 1965, no. 2 , p. 222.

3. J. T. Davies \& J. M. Vaughan, Astrophys. J., v. 137, 1963, p. 1302.

4. V. J. Bell, P. A. C. Buckler \& I. C. Pull, U. K. Atomic Energy Authority Report H.M.S.O. (Atomic Energy Establishment, Winfrith), 1963.

5. J. L. Cook \& D. Elliot, Austral. J. Appl. Sci., v. 11, 1960, pp. 16-32. MR 22 \#6130.

6. D. G. Hummer, Mem. Roy. Astronom. Soc., v. 70, 1965, pp. 1-31. MR 34 \#928.

7. J. B. Rosser, Theory and A pplication of $\int_{0}^{z} e^{-x^{2}} d x$ and $\int_{0}^{z} e^{-\rho 2 y^{2}} d y \int_{0}^{y} e^{-x^{2}} d x$. Part I: Methods of Computation, Mapleton House, Brooklyn, N. Y., 1948. MR 10, 267.

8. H. E. SAlzer, $M T A C$, v. 5, 1951, pp. 67-70. MR 13, 989.

9. O. N. Strand, Math. Comp., v. 19, 1965, pp. 127-129. MR $30 \# 694$.

10. V. N. Faddeyeva \& N. M. Terent'ev, Tables of Values of the Function $w(z)=$ $e^{-z^{2}}\left(1+2 i \pi^{-1 / 2} \int_{0}^{z} e^{t^{2}} d t\right)$ for Complex Argument, translated from the Russian, Pergamon Press, Oxford, 1961. MR 22 \#12740.

11. B. D. Fried \& S. D. Conte, The Plasma Dispersion Function, Academic Press, New York, 1961. MR 24 \#B1958.

12. P. C. Clemmow \& C. M. Munford, Philos. Trans. Roy. Soc. London Ser. A, v. 245, 1952, pp. 189-211. MR 14,500.

13. K. A. Karpov, Tables of the Function $w(z)=e^{-z^{2}} \int_{0}^{z} e^{x 2} d x$ in the Complex Domain, translated from the Russian, Pergamon Press, New York, 1965. MR 30 \#4992.

14. E. T. Goodwin, Proc. Cambridge Philos. Soc., v. 45, 1949, pp. 241-245. MR 10, 575.

15. M. Abramowitz \& I. A. Stegun, (EDitors), Handbook of Mathematical Functions, with Formulas, Graphs and Mathematical Tables, National Bureau of Standards, Applied Math. Series, No. 55, U. S. Government Printing Office, Washington, D. C., 1964. MR 29 \#4914.

16. P. C. Rosenbloom \& D. V. Widder, Trans. Amer. Math. Soc., v. 92, 1959, pp. 220-226.

MR 21 \#5845.

17. A. Reichel, J. Austral. Math. Soc., v. 4, 1964, pp. 476-488. MR 30 \#007.

18. C. Chiarella \& A. Reichel, Monthly Notices Roy. Astronom. Soc., 134, pp. 83-86.

19. Y. L. Luke, Integrals of Bessel Functions, McGraw-Hill, New York, 1962. MR 25 \#5198. 